elements have been made. Table I shows the element irradiated and the activities found. Some of the activities have been investigated or discussed earlier..$^{-5}$ Experiments are under way to study short-lived isomeric states in some heavier elements, and also to detect short-lived $\alpha$-decaying nuclides. In the latter case a thin layer of $\mathrm{ZnS}$ phosphor coated on Plexiglas has been used to discriminate $\alpha$ from $\beta$ and $\gamma$ pulses.

Experiments will be carried out with more channels in the analyzer to facilitate resolution of the decay

${ }^{1}$ R. K. Sheline, Phys. Rev. 87, 557 (1952).

2 T. Lauritsen, Ann. Revs. Nuclear Sci. 1, 85 (1952).

3 Glass, Jensen, and Richardson, Phys. Rev. 90, 320 (1953).

${ }^{4}$ W. M. Martin and S. W. Breckon, Can. J. Phys. 30, 643 (1952).

${ }^{5}$ Hollander, Perlman, and Seaborg, Revs. Modern Phys. 25, 469 (1953). curves and also perhaps with scintillation counters in coincidence.

Mass assignments will be made mainly by the study of excitation curves. Properties of the radiation will be studied by pulse-height analysis of the crystal pulses at different times after the cyclotron pulse.

The suggested assignments in Table $I$ are from a simple consideration of preferred reaction types and estimates of thresholds, with some mass values obtained from the table of Metropolis and Reitwiesner. ${ }^{6}$ It is probable that several corrections will have to be made as experiments proceed.

${ }^{6}$ N. Metropolis and G. Reitwiesner, Report NP-1980 (unpublished).

\title{
Energy Level Displacements in Pi-Mesonic Atoms
}

\author{
S. DeSER, Institute for Advanced Study, Princeton, New Jersey, \\ M. L. Goldberger, Institute for Nuclear Studies, and Department of Physics, University of Chicago, Chicago, Illinois,
}

K. Baumann, Physikalisches Institut, Universität Wien, Austria,

AND

W. ThIrring, Physikalisches Institut, Bern, Switzerland

(Received July 26, 1954)

\begin{abstract}
The energy level shifts and level widths of the $s$ states of the $\pi$-mesonic atoms are discussed. The discussion is limited to fairly light nuclei. On the basis of Orear's determination of the scattering lengths for meson-nucleon scattering, semiquantitative predictions are made. It is pointed out that even a knowledge of the algebraic sign of the level shift would be of value.
\end{abstract}

\section{INTRODUCTION}

$S^{\mathrm{E}}$ EVERAL years ago $^{1}$ the low-energy properties of the $\pi^{-}-p, \pi^{0}-n$ system were discussed on the basis of a formalism closely related to that of Wigner and Eisenbud. ${ }^{2}$ The system was treated as a two-channel nuclear reaction (taking into account only $s$ state interaction, which we shall also do here) with the boundary conditions at the surface of a sphere of a radius, the meson Compton wavelength being specified in terms of three real energy-dependent parameters. The only unusual feature in the calculation is the treatment of the capture of a negative pion from a bound $K$ orbit. $^{3}$

1 E. Fermi and M. L. Goldberger (unpublished); a short account of the work appears in Phys. Rev. 83, 239(A) (1951). An essentially equivalent derivation has recently been independently given by G. C. Wick (private communication). We are indebted to Prof. Wick for informing us of his results.

2 E. P. Wigner and L. Eisenbud, Phys. Rev. 72, 29 (1947).

3 The usual formulation of reaction theory deals with scattering rather than bound states. The necessary formal extension of the theory was later given independently by J. B. Ehrmann, Phys. Rev. 81, 412 (1951). Our problem is much simpler in that the small level shift and level width enables us to approximate the Coulomb functions in a simple way.
There appear quite naturally in the calculation the shift of the Bohr level associated with the pion-proton interaction and the width of the level due to the capture process. It is the purpose of the present note to deduce the level shift and level width in mesonic atoms in a more elementary and to a certain extent a less phenomenological manner than was done previously ${ }^{1}$ and further to discuss briefly the possibilities of comparison with experiment.

\section{DERIVATION OF THE LEVEL SHIFT AND LEVEL WIDTH}

Before taking up the details of the calculation it is expedient to discuss the rather peculiar role played by the Coulomb field in the mesonic atom problem as well as in the ordinary low-energy scattering. The Coulomb force is of course essential for the very existence of mesonic atoms and its influence on the low-energy scattering is quite marked in so far as the interpretation of the data is concerned. On the other hand, provided that the nuclear charge is low (exactly how low will appear later) the Coulomb field may be treated very 
simply, and in the scattering problem may be almost ignored since it scarcely influences even the zero energy scattering lengths, in contradistinction to the familiar proton-proton situation. There are two reasons for this: (1) The relevant parameter in the expansion of the reciprocal of the scattering length in terms of zerocharge quantities is the ratio of the range of the mesonnucleon interaction divided by one-half of the mesonic Bohr radius ${ }^{4}$ and this is a number of the order of $2 Z / 137$ as compared to the nuclear case where it is about $1 / 10$; and (2) the meson nucleon scattering lengths are small compared to the force range and one is not faced with a near resonance at zero energy which greatly magnifies the Coulomb corrections to $1 /$ (scattering length) in the $p$ - $p$ problem. Because of the second circumstance we can tolerate quite large values of $Z$, say up to about 30 . The capture rate for elements heavier than about oxygen is so rapid (from $s$ states) that this restriction is unimportant.

We now proceed to the actual calculation. We write the Schrödinger equation for the complete system as

$$
(K+C+V) \Psi=E \Psi,
$$

where $K$ is the kinetic energy operator which is taken to include the $n-p$ and $\pi^{-}-\pi^{0}$ mass differences, $C$ is the Coulomb interaction, and $V$ is the pion-nucleon interaction. (We consider first the $\pi^{-}-p$ system and will later generalize to the case of a nucleus.) We do not imply that $V$ is anything like a point potential in configuration space; it is necessary to assume only that it is effectively a short-range interaction. We choose as the zero of energy the energy of a negative pion and a proton infinitely far apart. Thus, because of the mass differences, even negative energies down to $-\left[M_{p}-M_{n}+M_{\pi^{-}}-M_{\pi^{0}}\right] \approx-3.6 \mathrm{Mev}$ are not stationary states. We ask now for the energy level shift due to $V$ of a negative pion bound in a $K$ orbit of energy $E_{0}$. If $\Psi$ is the complete wave function and $\Psi_{0}$ that of the stationary Bohr orbit which exists if $V$ is zero, we may write

$$
\begin{aligned}
\Psi & =\Psi_{0}+\left(1-P_{0}\right) \Psi \\
& =\left[1+\frac{1}{E-K-C-\left(1-P_{0}\right) V}\left(1-P_{0}\right) V\right] \Psi_{0},
\end{aligned}
$$

where $P_{0}$ is the projection operator onto the state $\Psi_{0}$ and we have chosen the normalization so that $\left(\Psi_{0}, \Psi\right)=1$. (We imagine the system to be enclosed in a large box, as usual.) From Eq. (1) and the equation satisfied by $\Psi_{0}$ it follows immediately that

$$
\begin{array}{r}
\delta E=E-E_{0}=\left(\Psi_{0},\left[V+V \frac{1}{E-K-C-\left(1-P_{0}\right) V}\right.\right. \\
\left.\left.\times\left(1-P_{0}\right) V\right] \Psi_{0}\right) .
\end{array}
$$

${ }^{4}$ G. F. Chew and M. L. Goldberger, Phys. Rev. 75, 1637 (1949).
This expression, which is exact, is to be compared to the matrix element describing the forward scattering of positive energy pions by protons (neglecting Coulomb effects), namely,

$$
\begin{aligned}
& R_{00} \equiv\left(\chi_{0}, R\left(E_{+}\right) \chi_{0}\right) \\
& \quad=\left(\chi_{0},\left[V+V^{\circ} \frac{1}{E_{+}-K-V+i \epsilon} V\right] \chi_{0}\right),
\end{aligned}
$$

where $\chi_{0}$ represents a plane wave state corresponding to the relative motion of a negative pion and a proton with the positive energy $E_{+}$, normalized to unity in a box of volume $\Omega$. We wish to see to what extent the energy shift, Eq. (3), may be expressed in terms of $R_{00}$. If we were to neglect the Coulomb field inside the square brackets of Eq. (3), which means setting $C$ and $P_{0}$ equal to zero, we would have very nearly the expectation value of the operator $R$ occurring in Eq. (4). There are two differences, namely, the numerical value of the energies, and the presence of the outgoing wave instruction, " $i \epsilon$," in Eq. (4). As to the first we shall set $E_{+}=0$ so as to deal eventually with conventional zero energy scattering lengths and then assume that the energy variation of the operator in going from zero to about $-3200 \mathrm{ev}$ (in the case of $\mathrm{H}$, and about $Z^{2} \times 3700$ ev for a nucleus) is unimportant. If what is essentially the real part of the operator $R$ is chosen by using a principal value rather than the " $i \epsilon$," the second of the above differences disappears, since in our quasi-continuum situation (which becomes a true continuum as $\Omega \rightarrow \infty$ ) such principal values are implied in Eq. (3). More precisely, the real part of $R_{00}$ is obtained by using principal values instead of the " $i \epsilon$ " conditions. This feature is not changed by the presence of $\Psi_{0}$ in Eq. (4) rather than $\chi_{0}$. We may write then finally

$$
\delta E \simeq \operatorname{Re}\left(\Psi_{0}, R(0) \Psi_{0}\right) .
$$

If we now make use of the assumed short range of $V$ to replace the wave function by their values at the origin, we may write

$$
\delta E \simeq \operatorname{Re}\left|\Psi_{0}(0)\right|{ }^{2} \Omega\left(\chi_{0}, R(0) \chi_{0}\right) .
$$

If we were to simply insert $R(0)$ with the " $i \epsilon$ " included in place of the bracket in Eq. (3), we would obtain Eq. (6) without the instruction to take the real part, and we obtain an imaginary contribution to the level shift which is, of course, interpreted as the level width. To save writing we shall use such complex energy shifts. We may relate $\left(\chi_{0}, R(0) \chi_{0}\right)$ to the zero energy scattering length, $a\left(\pi^{-}\right)$, for ordinary (nonexchange) scattering of negative pions by protons according to

$$
\Omega\left(\chi_{0}, R(0) \chi_{0}\right)=-(2 \pi / \bar{\mu}) a\left(\pi^{-}\right),
$$

where $\bar{\mu}$ is the reduced mass of the pion-proton system. The total level shift (real and imaginary parts) is 
given by

$$
\delta E=-(2 \pi / \bar{\mu})\left|\Psi_{0}(0)\right|^{2} a\left(\pi^{-}\right) .
$$

In the next section we shall compare these predictions with experiment.

\section{COMPARISON WITH EXPERIMENT}

To the zeroth order in the mass differences, $a\left(\pi^{-}\right)$may be expressed directly in terms of the scattering lengths for states of isotopic spin $\frac{1}{2}$ and $\frac{3}{2}$ which we shall call $a_{1}$ and $a_{3}$ respectively. We have

$$
a\left(\pi^{-}\right)=\frac{1}{3}\left(2 a_{1}+a_{3}\right) .
$$

In this approximation $a\left(\pi^{-}\right)$is real, since there is no decay if the mass difference vanishes. Inserting the value of $\left|\Psi_{0}(0)\right|^{2}$, namely $1 / \pi r_{B}{ }^{3}$ with $r_{B}$ the mesonic Bohr radius $\left(1 / \bar{\mu} e^{2}\right)$, we obtain for the level shift in hydrogen

$$
\delta E / E_{0}=-4\left(2 a_{1}+a_{3}\right) / 3 r_{B},
$$

where $E_{0}$ is the Bohr energy, $e^{2} / 2 r_{B}$. For a nucleus of charge $Z$, assuming the effects of the various nucleons are simply additive, and recalling that the $\pi^{-}-n$ scattering length is $a_{3}$, we find

$$
\frac{\delta E}{E_{Z}}=-\frac{4 Z}{r_{B}}\left[\begin{array}{l}
2 \\
-Z \\
3
\end{array} a_{1}+\frac{3 N+Z}{3} a_{3}\right] \text {. }
$$

$E_{Z}$ is in Eq. (11) the Bohr energy appropriate to a nucleus of charge $Z$. Taking the values of $a_{1}$ and $a_{3}$ given by Orear, ${ }^{5}$ namely $a_{1}=0.16 / \mu, a_{3}=-0.11 / \mu$, we find for hydrogen $\delta E / E_{0} \sim-1.8 \times 10^{-3}$ and $\delta E \sim-6 \mathrm{ev}$. For a nucleus with $N=Z, \delta E / E_{Z} \sim+Z^{2} / 850$. We must expect our approximations on the Coulomb field to fail when $\delta E / E_{Z} \sim 1$ so that we restrict our attention to $Z \ll 30$. A particular case of interest is $\mathrm{Be}^{9}$, for which we obtain $\delta E \sim+1850 \mathrm{ev}$. The corrections due to finite mass differences cannot be calculated without a specific model, but the considerations of the capture process to be treated immediately below indicate that they are small. There is of course also a level shift due to the extended electric charge in a nucleus other than hydrogen but this is very small compared to those considered here, for light nuclei.

The imaginary part of the level shift, i.e., the level width, involves a knowledge of the imaginary part of $a\left(\pi^{-}\right)$at zero energy. This quantity may be computed directly from the unitarity condition on the $R$ matrix, ${ }^{6}$ namely

$$
-\operatorname{Im} R_{a a}=\pi \sum_{b}\left|R_{b a}\right|^{2} \delta\left(E_{b}-E_{a}\right),
$$

applied to a state $a$ representing a zero kinetic energy pion and proton. The only states $b$ that make a non-

\footnotetext{
5 Jay Orear, Phys. Rev. 96, 176 (1954).

${ }^{6}$ B. Lippmann and J. Schwinger, Phys. Rev. 79, 469 (1950).
}

vanishing contribution are those associated with the capture process and we find from Eq. (12), using Eq. (7),

$$
\operatorname{Im} a\left(\pi^{-}\right)=q_{i} \sigma_{c} /(4 \pi)=q\left|a\left(\pi^{0} \leftarrow \pi^{-}\right)\right|^{2},
$$

where $q$ is the relative momentum of the $\pi^{0}-n$ system, related to the mass difference by $q^{2} / 2 \bar{\mu}=\delta M$, and $q_{i} \sigma_{c}$ is the product of the (zero) momentum of the incident pion and the charge exchange cross section at zero energy; $a\left(\pi^{0} \leftarrow \pi^{-}\right)$is the charge exchange scattering length. From Eq. (8), then, we find an imaginary contribution to the level shift, namely,

$$
\operatorname{Im}\left(\delta E / E_{0}\right)=-4\left(q r_{B}\right)\left|a\left(\pi^{0} \leftarrow \pi^{-}\right) / r_{B}\right|^{2} .
$$

To zero order in $q, a\left(\pi^{0} \leftarrow \pi^{-}\right)$is given by

$$
a\left(\pi^{0} \leftarrow \pi^{-}\right)=\frac{1}{3}(2)^{\frac{1}{2}}\left(a_{1}-a_{3}\right),
$$

and thus we find from Eq. (14) a level width $\Gamma$ $(\Gamma=-\operatorname{Im} \delta E)$,

$$
\Gamma / E_{0}=(8 / 9)\left(q r_{B}\right)\left|\left(a_{1}-a_{3}\right) / r_{B}\right|^{2} .
$$

For a nucleus of charge $Z$ (since only the protons contribute to the capture process considered here),

$$
\Gamma / E_{Z}=(8 / 9) Z^{2}\left(q r_{B}\right)\left|\left(a_{1}-a_{3}\right) / r_{B}\right|^{2} .
$$

In hydrogen, $\Gamma \sim 0.3 \mathrm{ev}$, whereas in $B e$ it is about $77 \mathrm{ev}$. Thus in both cases the level width is much smaller than the level shift and consequently the expansion in powers of $q r_{B}$ which we have used should be quite accurate. This parameter is about $\frac{1}{4}$ and the correction terms to both the level shift and the width are proportional to $\left(q r_{B}\right)^{2}$.

\section{CONCLUSIONS}

We shall not enter into an exhaustive discussion of the various effects which can contribute to the level width and level shift which have not been considered here. For light elements the effect we have been discussing would appear to be the most important. There may be significant contributions to the width due to star formation, but the level shift is probably not greatly affected. The only measurements of the level shift carried out to date are some very preliminary ones at Carnegie $\mathrm{Tech}^{7}$ on $\mathrm{Be}^{9}$ which show effects of the same order of magnitude as those considered here.

It would be of considerable interest to know even the algebraic sign of the level shift in several elements, for this alone would enable us to draw some conclusions about the signs of the scattering lengths. For example, Orear's assignment yields always a positive $\delta E$ (i.e., less binding, a repulsive interaction) whereas any assignment which makes $a_{1}$ and $a_{3}$ both positive leads to tighter binding, negative $\delta E$ 's.

\footnotetext{
${ }^{7}$ Private communication from Professor de Benedetti to Professor Fermi.
} 